Article

\title{
Evaluation of Predictors of Biochemical Recurrence in Prostate Cancer Patients, as Detected by ${ }^{68}$ Ga-PSMA PET/CT
}

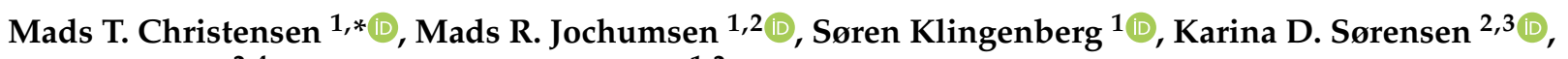 \\ Michael Borre ${ }^{2,4}$ and Kirsten Bouchelouche ${ }^{1,2}$ \\ 1 Department of Nuclear Medicine, PET-Centre, Aarhus University Hospital, 8200 Aarhus, Denmark; \\ madsjoch@rm.dk (M.R.J.); SOEKLI@rm.dk (S.K.); Kirsten.Bouchelouche@auh.rm.dk (K.B.) \\ 2 Department of Clinical Medicine, Aarhus University, 8000 Aarhus, Denmark; kdso@clin.au.dk (K.D.S.); \\ borre@clin.au.dk (M.B.) \\ 3 Department of Molecular Medicine, Aarhus University Hospital, 8200 Aarhus, Denmark \\ 4 Department of Urology, Aarhus University Hospital, 8200 Aarhus, Denmark \\ * Correspondence: matrch@rm.dk; Tel.: +45-5050-2997; Fax: +45-7845-6264
}

check for

updates

Citation: Christensen, M.T.; Jochumsen, M.R.; Klingenberg, S.; Sørensen, K.D.; Borre, M.; Bouchelouche, K. Evaluation of Predictors of Biochemical Recurrence in Prostate Cancer Patients, as Detected by ${ }^{68}$ Ga-PSMA PET/CT. Diagnostics 2022, 12, 195. https:// doi.org/10.3390/diagnostics12010195

Academic Editor: Jochen Neuhaus

Received: 7 December 2021

Accepted: 11 January 2022

Published: 14 January 2022

Publisher's Note: MDPI stays neutral with regard to jurisdictional claims in published maps and institutional affiliations.

Copyright: (c) 2022 by the authors. Licensee MDPI, Basel, Switzerland. This article is an open access article distributed under the terms and conditions of the Creative Commons Attribution (CC BY) license (https:// creativecommons.org/licenses/by/ $4.0 /)$.

\begin{abstract}
Objectives: To explore the existence of new predictors of the ${ }^{68} \mathrm{Ga}$-Prostate-Specific Membrane Antigen (PSMA) PET/CT detection rate at biochemical recurrence (BCR) and to determine the detection rate of ${ }^{68} \mathrm{Ga}$-PSMA PET/CT dependent of prostate-specific antigen (PSA) levels. Materials and methods: In total, 189 PCa patients scanned with ${ }^{68}$ Ga-PSMA PET/CT for detection of BCR after curatively intended treatment with either radical prostatectomy $(n=153)$ or radiotherapy $(n=36)$ were included. Clinicopathological information at the time of diagnosis (PSA, clinical tumor-stage, International Society of Urological Pathology Grade Group and whether ${ }^{68}$ Ga-PSMA PET/CT was used for primary staging), treatment (RT/RP and histopathology of the prostatectomies), and pre-PET PSA were collected from medical records. Results: Of the $189{ }^{68} \mathrm{Ga}-\mathrm{PSMA}$ PET/CT scans, 103 (54.5\%) were positive for BCR of PCa. No significant coherency was observed between detection rate and any clinicopathological variables at diagnosis. Detection rates significantly increased with rising PSA: $<0.5 \mathrm{ng} / \mathrm{mL}=28 \%, 0.5 \leq 1 \mathrm{ng} / \mathrm{mL}=39 \%, 1 \leq 2 \mathrm{ng} / \mathrm{mL}=64 \%, 2 \leq 5 \mathrm{ng} / \mathrm{mL}=87.5 \%$ and $\geq 5 \mathrm{ng} / \mathrm{mL}=97 \%$. Conclusions: The detection rate of PCa recurrence was strongly dependent of pre-PET PSA levels. None of the additional clinical variables acquired during primary staging, prostatectomy pathology reports, nor primary staging imaging modality affected the detection rate.
\end{abstract}

Keywords: prostate cancer; ${ }^{68}$ Ga-Prostate-Specific Membrane Antigen PET/CT; ${ }^{68}$ Ga-PSMA; biochemical recurrence; detection rate; predictors

\section{Introduction}

Prostate cancer $(\mathrm{PCa})$ is the second most commonly diagnosed male cancer, accounting for 358.989 registered cases of death in 2018, and thereby being the fifth leading cause of cancer death among men worldwide [1]. The options for curative intended treatment of $\mathrm{PCa}$ are either radical prostatectomy $(\mathrm{RP})$ or radiotherapy (RT). As a result of different treatment options for the primary disease, biochemical recurrence (BCR) is, according to the standard care in Denmark, defined by PSA $\geq 0.2 \mathrm{ng} / \mathrm{mL}$ post RP and by PSA reaching nadir $+2.0 \mathrm{ng} / \mathrm{mL}$ post RT [2]. Relapse detection of BCR has become a well-established indication for ${ }^{68} \mathrm{Ga}$-Prostate-Specific Membrane Antigen (PSMA)-PET/CT [3]. Several PSMA ligands have been developed, with ${ }^{68}$ Ga-PSMA-11(Glu-CO-Lys(Ahx)-HBED-CC) being one of the most commonly used compounds [4].

${ }^{68} \mathrm{Ga}-\mathrm{PSMA}$ PET/CT is a commonly used modality in patients with BCR, as it has been shown to be superior to conventional diagnostic imaging in locating recurrent disease [5-8]. This advantage is especially noticeable in cases with low PSA values $(<0.5 \mathrm{ng} / \mathrm{mL})[9,10]$. In the case of BCR, it is crucial to locate the anatomical site of recurrence regarding treatment options where ${ }^{68} \mathrm{Ga}-\mathrm{PSMA}$ PET/CT has shown to influence the management of patients 
with recurrent PCa [11]. While an association has been found between increasing PSA levels and the detection rate of ${ }^{68} \mathrm{Ga}$-PSMA PET/CT in recurrent disease, most studies did not report clinical data from the time of diagnosis, and hence no additional predictors of detection rate have been described. In the present cohort, we have access to imaging and clinical data from both the primary staging, treatment and BCR.

Hence, the aim of this retrospective study was to investigate whether any of these reported clinicopathological variables could serve as possible predictors of the detection rate of ${ }^{68} \mathrm{Ga}$-PSMA PET/CT in patients with recurrent disease. An additional aim of the study was to compare the ${ }^{68} \mathrm{Ga}-\mathrm{PSMA}$ PET/CT detection rates dependent on pre-PET PSA levels with results from the existing literature.

\section{Materials and Methods}

\subsection{Patient Population}

From April 2016 through March 2019, a total of $1101{ }^{68}$ Ga-PSMA PET/CT scans were conducted at the Department of Nuclear Medicine and PET-Centre, Aarhus University Hospital, Denmark. PCa patients scanned with ${ }^{68} \mathrm{Ga}-\mathrm{PSMA}$ PET/CT for detection of BCR after curatively intended treatment with either RP or RT were included. Patients referred for primary staging or with previously proven dissemination were excluded. Of the 1101 patients, a total of 189 (17.1\%) met the criteria (Figure 1).

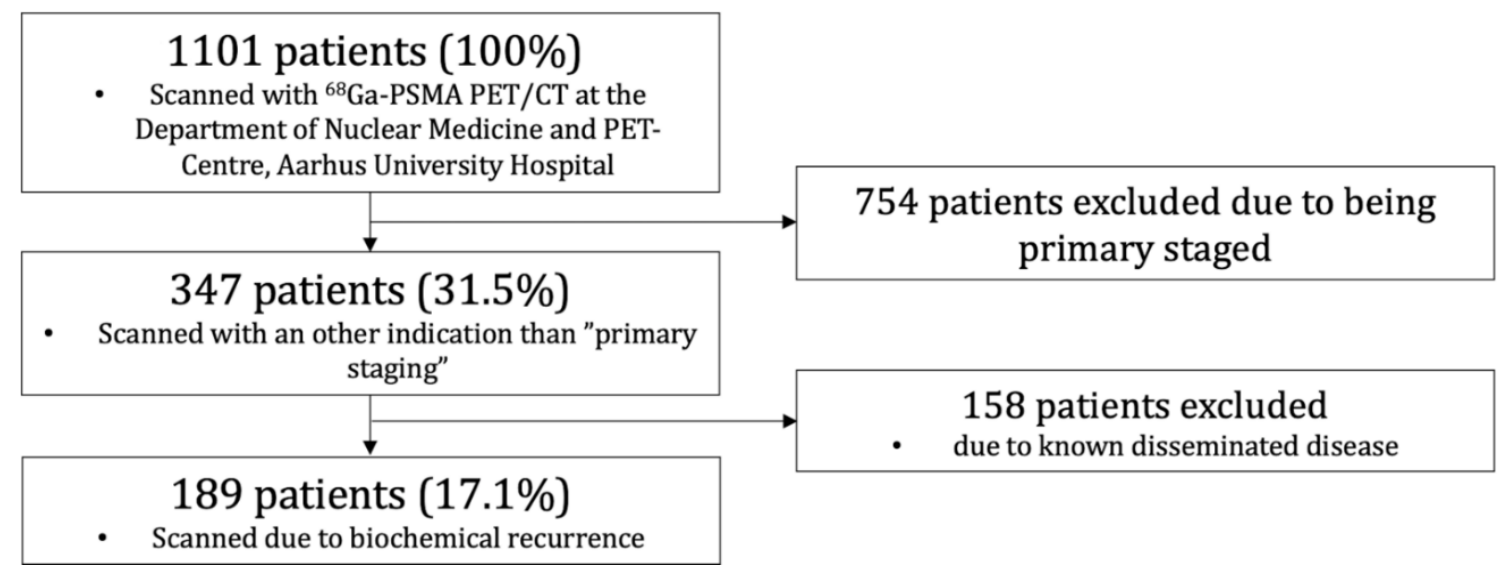

Figure 1. Study flow for ${ }^{68} \mathrm{Ga}$-PSMA PET/CT of patients with biochemical recurrence.

Data was available through medical records and collected and managed using REDCap electronic data capture tools, hosted at Aarhus University [12,13]. Clinicopathological information at the time of diagnosis (PSA (latest before treatment), clinical tumor (cT)-stage, Gleason Score, International Society of Urological Pathology (ISUP) Grade Group [14], and whether ${ }^{68}$ Ga-PSMA PET/CT was used for primary staging), information concerning their treatment (RP/RT and pathology in case of RP) and lastly, clinical information at the time of recurrent disease (PSA at scan, results and location of recurrent disease) were attained. The ISUP Grade Group is defined as; Group 1 = Gleason Score 6, Group 2 = Gleason Score 7 $(3+4)$, Group $3=$ Gleason Score $7(4+3)$, Group $4=$ Gleason Score 8, and Group $5=$ Gleason Score 9-10 [14]. As some patients were diagnosed before the establishment of the electronical medical records and some were referred from other regions of Denmark (thus resulting in inaccessible records), information regarding certain variables, especially about the time of diagnosis, was unavailable in some patients. The time from primary diagnosis to first diagnostic imaging varies greatly in this cohort, as some patients are examined in fast track while low risk patients could have gone in active surveillance for years before the first diagnostic imaging.

This retrospective study was approved by the institutional review board (Central Denmark Region Committees on Health Research Ethics). 


\section{2. ${ }^{68} \mathrm{Ga}$-PSMA PET/CT}

The production of ${ }^{68} \mathrm{Ga}-\mathrm{PSMA}-11$ ( ${ }^{68} \mathrm{Ga}-\mathrm{Glu}-\mathrm{CO}-\mathrm{Lys}(\mathrm{Ahx})$-HBED-CC) was produced according to good manufacturing practice (GMP) and QC following the national Danish regulations. All ${ }^{68} \mathrm{Ga}-\mathrm{PSMA}$ PET/CT scans were performed according to EANM and SNMMI guidelines [15]. The patients did not fast before the scan, but were well hydrated before the study and during the tracer uptake time (e.g., oral intake of $500 \mathrm{~mL}$ of water during a $2 \mathrm{~h}$ period prior to acquisition). The patients received an intravenous bolus injection of $2.14 \mathrm{MBq}{ }^{68} \mathrm{Ga}$-PSMA-11 per kilogram bodyweight approximately $60 \mathrm{~min}$ before the scan. The injection of the tracer was followed by flushing of the syringe with at least the same volume of saline $(\mathrm{NaCl} 0.9 \%)$ to maximize use of dispensed activity. ${ }^{68} \mathrm{Ga}$-PSMA PET/CT scans were performed with low-dose CT for anatomical localization and attenuation correction. Just before the static PET/CT scan, the patients emptied their urinary bladder. All scans were performed with a Siemens Biograph TruePoint PET/CT scanner (Siemens, Erlangen, Germany). The patients were positioned with both arms elevated above the head, as tolerated by the patients. The patients were PET/CT scanned from vertex cranii to mid-thigh. All available corrections (attenuation, scatter and PointSpread Function) were applied in the image reconstruction using the TrueX reconstruction algorithm (4 iterations and 21 subsets) and a $3 \mathrm{~mm}$ Gaussian post-filter (XYZ) and voxel size of $2 \times 2 \times 2 \mathrm{~mm}$.

\subsection{Image Analysis}

The analyses of all scans were performed by experienced, board certified specialists in nuclear medicine, in most cases by a single reader with many years of experience in prostate cancer PET imaging. A second evaluation was performed by an experienced specialist at the weekly prostate cancer multidisciplinary team conference. In cases of disagreement, a consensus was made between the experienced specialists. Image analysis was performed using Hybrid Viewer (HERMES Medical Solutions AB, Stockholm, Sweden). The scan results were defined as "positive", "negative" or "equivocal". Positive was defined as visually avid focal tracer uptake not associated with physiological uptake and higher than adjacent tissue, and hence suspicious of malignancy, these criteria are in accordance with Prostate Cancer Molecular Imaging Standardized Evaluation (PROMISE) guidelines [16] and with the major publications on this topic $[15,17,18]$. Equivocal was defined as cases with increased focal tracer uptake where it was not possible to distinguished between a benign or malignant condition, which in most cases leads to a follow-up biopsy or MRI scan. Negative was defined as no suspicious tracer uptake. Pathological ${ }^{68}$ Ga-PSMA uptake in the prostatic bed was defined as "local", uptake in pelvic lymph nodes below the common iliac artery bifurcation was defined as lymph node metastases (LNMs), and thus "N1" disease (PROMISE-criteria [16]), whereas extra pelvic LNMs were defined as "M1a". Lesions proposing bone metastases and visceral metastases were defined as "M1b" and "M1c", respectively.

The suspicious lesions on ${ }^{68} \mathrm{Ga}$-PSMAPET/CT were biopsied, when possible and clinically relevant, for histopathological confirmation of the findings as reference standard. The involved specialists were aware of the possible pitfalls when reading the PSMA PET/CT scans [19].

\subsection{Statistical Analysis}

Statistical analyses were performed using Stata 16.1. The variables were summarized using descriptive statistics. Continuous variables were examined for normal distribution using $\mathrm{Q}-\mathrm{Q}$ plots. If the continuous variables were not normally distributed, they were presented as median (range). Detection rate was defined as the proportion of patients with positive ${ }^{68} \mathrm{Ga}$-PSMA PET/CT scans in comparison to patients with negative or equivocal results. Equivocal results were grouped with the negative scans due to their uncertainty and inability to distinguish between benign and malignant lesions, thereby not being able to define the lesions as "positive". Detection rates were compared in different analyses 
with stratification for different variables using $\chi^{2}$-tests with a significance level of $p<0.05$ using the actual numbers for positive and negative scans.

\section{Results}

\subsection{Patient Summary}

Of the $189^{68}$ Ga-PSMA PET/CT recurrence scans, a total of 103 (54.5\%) were positive, $12(6.3 \%)$ were equivocal and grouped with the $74(39.2 \%)$ negative scans. Therefore, a total of $86(45.5 \%)$ scans were defined as negative in the analysis.

Regarding treatment prior to the ${ }^{68} \mathrm{Ga}$-PSMA PET /CT scan, 153 (81\%) underwent RP and $36(19 \%)$ underwent RT with a detection rate of $44.4 \%$ and $97.2 \%$ in the RP and RT groups, respectively (Table 1 ).

Table 1. Patient summary (All patients).

\begin{tabular}{cccc}
\hline Characteristic & All Patients, $\boldsymbol{n = 1 8 9}$ & Positive Scans, $\boldsymbol{n = 1 0 3}$ & Negative Scans, $\boldsymbol{n}=\mathbf{8 6}$ \\
\hline Age at scan, median (range), $\mathbf{~}$ & $69.2(50.9-80.5)$ & $68.1(52.3-80.5)$ & $69.7(50.9-79.2)$ \\
\hline $\begin{array}{c}\text { PSA before treatment, median } \\
\text { (range), } \mathbf{n g} / \mathbf{m L}\end{array}$ & $10.5(0.9-183)$ & $10.8(3.2-183)$ & $10.3(0.9-138)$ \\
Unavailable $(n)$ & 5 & 3 & 2 \\
\hline Initial therapy $(n)$ & & & 85 \\
$R P$ & 153 & 35 & 1 \\
$R T$ & 36 & 68 & \\
\hline
\end{tabular}

\section{2. ${ }^{68} \mathrm{Ga}-P S M A$ PET/CT Detection Rate and Tumor Characteristics during Primary Staging}

PET detection rate of the relapse was compared when stratifying for different variables acquired during primary staging (Table 2). Analyses were performed when taking the latest PSA before treatment $(<10,10-20,>20 \mathrm{ng} / \mathrm{mL})$, cT-stage ( $\leq \mathrm{cT} 2 \mathrm{a}, \mathrm{cT} 2 \mathrm{~b}, \geq \mathrm{cT} 2 \mathrm{c})$, ISUP Grade Group and risk stratification (D'Amico classification [20]) individually into consideration. None of these variables affected the PET detection rate (Table 2) $(p>0.05)$.

Table 2. Tumor characteristics during primary staging (All patients).

\begin{tabular}{|c|c|c|c|}
\hline Characteristic & All Patients, $n=189$ & PET Positive Results, $n,(\%)$ & $x^{2}$ \\
\hline \multicolumn{4}{|c|}{$\begin{array}{l}\text { PSA before treatment, } \\
\mathrm{ng} / \mathrm{mL}\end{array}$} \\
\hline$<10$ & 86 & $46(53.5)$ & \multirow{4}{*}{$p=0.948$} \\
\hline $10-20$ & 57 & $32(56.1)$ & \\
\hline$>20$ & 41 & $22(53.7)$ & \\
\hline Unavailable $(n)$ & 5 & - & \\
\hline \multicolumn{4}{|c|}{ cT-stage, prior treatment } \\
\hline$<\mathrm{cT} 2 \mathrm{a}$ & 70 & $35(50)$ & \multirow{4}{*}{$p=0.667$} \\
\hline $\mathrm{cT} 2 \mathrm{~b}$ & 10 & $6(60)$ & \\
\hline$>\mathrm{cT} 2 \mathrm{c}$ & 38 & $22(57.9)$ & \\
\hline Unknown & 71 & - & \\
\hline \multicolumn{4}{|c|}{$\begin{array}{l}\text { ISUP Grade Group, prior } \\
\text { treatment }\end{array}$} \\
\hline 1 & 30 & $18(60)$ & \multirow{6}{*}{$p=0.832$} \\
\hline 2 & 58 & $30(51.7)$ & \\
\hline 3 & 31 & $17(54.8)$ & \\
\hline 4 & 45 & $26(57.8)$ & \\
\hline 5 & 20 & $9(45)$ & \\
\hline Unknown & 5 & - & \\
\hline
\end{tabular}


Table 2. Cont.

\begin{tabular}{cccc}
\hline Characteristic & All Patients, $\boldsymbol{n = 1 8 9}$ & PET Positive Results, $\boldsymbol{n}, \mathbf{( \% )}$ & $\chi^{\mathbf{2}}$ \\
\hline Risk stratification & & & \\
(D'Amico) & 18 & $9(50)$ & \\
Low & 70 & $41(58.6)$ & \\
Intermediate & 95 & $49(51.6)$ & \\
High & 6 & - & \\
Unknown & & & \\
\hline
\end{tabular}

\section{3. ${ }^{68} \mathrm{Ga}-P S M A$ PET/CT Detection Rate in Patients Treated with RP}

In the 153 patients treated with RP, information regarding ISUP Grade Group, surgical margins and pathological tumor (pT)-stage was accessible in most patients (Table 3). The detection rate was independent of these pathological characteristics $(p>0.05)$.

Table 3. Patients initially treated with RP.

\begin{tabular}{cccc}
\hline & All RP Patients, $\boldsymbol{n = 1 5 3}$ & $\begin{array}{c}\text { PET-Positive Results, } \\
\boldsymbol{n}, \mathbf{( \% )}\end{array}$ & $\chi^{\mathbf{2}}$ \\
\hline $\begin{array}{c}\text { ISUP Grade Group, } \\
\text { prostatectomy }\end{array}$ & & & \\
1 & 10 & $7(70)$ & \\
2 & 55 & $22(40)$ & \\
3 & 38 & $18(47.4)$ & \\
4 & 22 & $9(40.9)$ & \\
5 & 23 & $10(43.5)$ & \\
Unknown & 5 & - & \\
Surgical margins & & & \\
Positive & 48 & $20(41.7)$ & \\
Negative & 101 & $47(46.5)$ & \\
Unknown & 4 & - & \\
pT-stage & & & \\
<pT2a & 5 & $1(20)$ & \\
pT2b & 2 & $64(44.1)$ & \\
>pT2c & 145 & - & \\
Unknown & 1 & & \\
\hline
\end{tabular}

Recategorizing pT-stage $(<$ pT3a and $\geq$ pT3a) and ISUP Grade Group $(<4$ and $\geq 4)$ did not affect the results $(p>0.05)$.

\subsection{Primary Staged Using ${ }^{68} \mathrm{Ga}-\mathrm{PSMA}$ PET/CT vs. Other}

Forty patients $(21.2 \%)$ had ${ }^{68} \mathrm{Ga}$-PSMA PET/CT conducted for primary staging purposes as well as having ${ }^{68} \mathrm{Ga}$-PSMA PET/CT conducted due to BCR. The rest of the patients $(n=149(78.8 \%))$ had a ${ }^{18} \mathrm{~F}$-choline-PET, bone scintigraphy and/or CT conducted or no imaging during their primary staging.

The detection rate was substantially lower for the patients with ${ }^{68} \mathrm{Ga}$-PSMA PET/CT for primary staging purposes $(35 \%)$ compared to patients with other or no imaging for primary staging $(59.7 \%)$. The patients with ${ }^{68} \mathrm{Ga}$-PSMA PET/CT staging had lower PSA levels as well, and after stratification for PSA, the impact of imaging modality for primary staging disappears.

\subsection{PSA-Level at Recurrent Disease}

PSA right before ${ }^{68} \mathrm{Ga}$-PSMA PET/CT at BCR was available in 187 patients $(98.9 \%)$. Median PSA at BCR was $0.62 \mathrm{ng} / \mathrm{mL}(0.1-127)$ for all 187 patients, whereas median PSA was $0.4 \mathrm{ng} / \mathrm{mL}(0.1-127)$ in the RP group and $4.9 \mathrm{ng} / \mathrm{mL}(0.1-124)$ in the RT group. Detection rate stratified by PSA is given in Table 4. A significant increase in detection rate was 
observed for all PSA intervals. Similar results were observed in the RP group, only with a small decrease in the detection rate (Table 5) compared to the joint patient group (RP $+\mathrm{RT}$ ). Not only an increase in detection rate was observed, disease was more likely to spread to multiple locations with increasing PSA as well (Figure 2).

Table 4. ${ }^{68}$ Ga-PSMA PET/CT detection rate dependent of PSA at recurrent disease, RP and RT combined.

\begin{tabular}{cccc}
\hline Stratification & $\boldsymbol{n}$ & $\begin{array}{c}\text { PET-Positive Results, } \\
\boldsymbol{n}(\mathbf{\%})\end{array}$ & $\boldsymbol{\chi}^{\mathbf{2}}$ \\
\hline All patients & 189 & $103(54.5)$ & \\
PSA ng/mL & & $23(28)$ & 0.01 \\
$<0.5$ & 82 & $9(39)$ & $16(64)$ \\
$0.5 \leq 1$ & 23 & $21(87.5)$ & \\
$1 \leq 2$ & 25 & $32(97)$ & \\
$2 \leq 5$ & 24 & - & \\
\hline 5 & 33 & 2 & \\
\hline
\end{tabular}

Table 5. ${ }^{68}$ Ga-PSMA PET/CT detection rate dependent of PSA at recurrent disease, RP only.

\begin{tabular}{cccc}
\hline Stratification & $\boldsymbol{n}$ & $\begin{array}{c}\text { PET-Positive Results, } \\
\boldsymbol{n}, \mathbf{( \% )}\end{array}$ & $\boldsymbol{\chi}^{\mathbf{2}}$ \\
\hline All patients & 153 & $68(44.4)$ & $p<0.01$ \\
PSA ng/mL & 79 & $21(26.6)$ & \\
$<0.5$ & 22 & $8(36.4)$ & $14(60.9)$ \\
$0.5 \leq 1$ & 23 & $9(75)$ & $15(93.8)$ \\
$1 \leq 2$ & 12 & - & \\
$2 \leq 5$ & 16 & 1 & \\
\hline Unknown & 1 & & \\
\hline
\end{tabular}

Detection Rate Stratified by PSA and Region

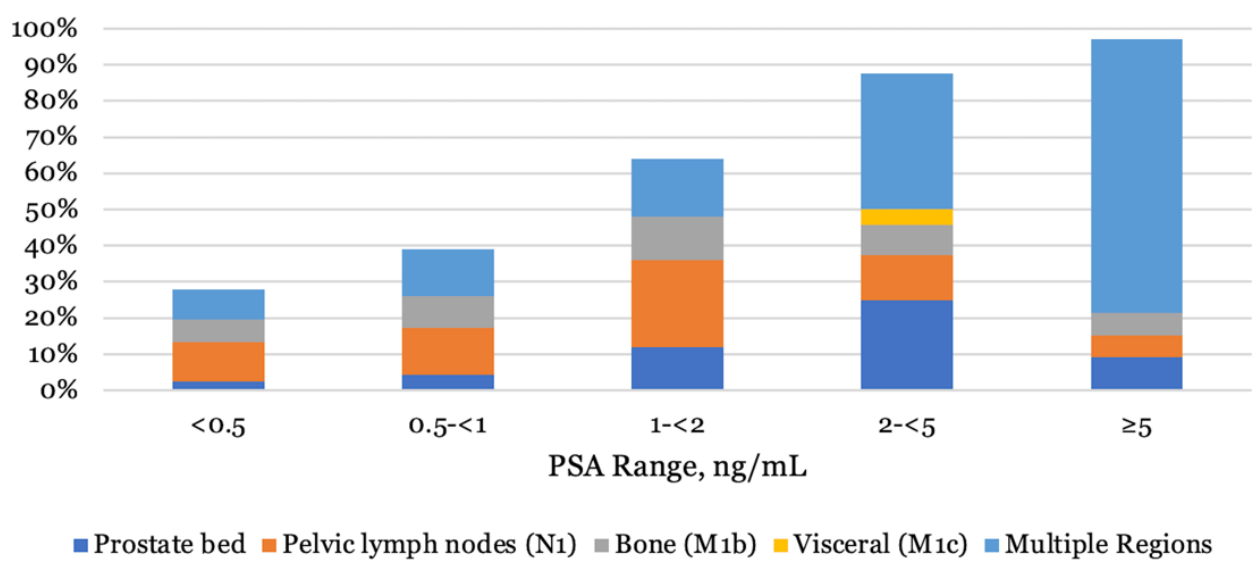

Figure 2. Proportion of patients with ${ }^{68} \mathrm{Ga}-\mathrm{PSMA}$ PET/CT positive findings, stratified by PSA and region in all patients $(n=189)$.

Analysis including RT patients only was not conducted due to the few numbers of patients and due to the fact that nearly all RT patients were in the $2 \leq 5$ or $\geq 5 \mathrm{ng} / \mathrm{mL}$ strata.

\section{Discussion}

The use of ${ }^{68}$ Ga-PSMA PET/CT in PCa has shown great potential in the clinical management of PCa, both for primary staging of high risk PCa and for detection of biochemical recurrence [21]. PSMA expression has been shown to be amplified in PCa with 
high preoperative PSA, tumor Gleason Score and advanced tumor stage [22]. In accordance with this, maximum standardized uptake value $\left(\mathrm{SUV}_{\max }\right)$ has been shown to correlate with ISUP Grade Group in the primary tumor [23-25]. Regarding recurrent disease, Vinsensia et al. [26] $(n=147)$ demonstrated a higher $S U V_{\max }$ in LNMs with high Gleason Scores $(\geq 8)$ than in intermediate and low grade PCa. While an association has been found between increasing PSA levels and the detection rate of ${ }^{68} \mathrm{Ga}-\mathrm{PSMA}$ PET/CT in recurrent disease, most studies did not report clinical data from the time of diagnosis, and hence no additional predictors of detection rate have been described.

The aim of this retrospective study was to investigate whether several variables like clinicopathological information at the time of diagnosis (PSA, clinical tumor-stage, International Society of Urological Pathology Grade Group and whether ${ }^{68}$ Ga-PSMA PET/CT was used for primary staging), treatment (RT/RP and histopathology of the prostatectomies), and pre-PET PSA could serve as possible predictors of the detection rate of ${ }^{68} \mathrm{Ga}-\mathrm{PSMA}$ PET/CT in patients with recurrent disease. The study showed no significant association between the detection rate of ${ }^{68} \mathrm{Ga}$-PSMA PET/CT at the time of recurrent disease and characteristics of the primary cancer at baseline or at RP. Similarly, having ${ }^{68} \mathrm{Ga}-\mathrm{PSMA}$ PET/CT conducted for primary staging did not seem to affect the detection rate of ${ }^{68} \mathrm{Ga}-$ PSMA PET/CT in BCR, when compared to primary staging with conventional imaging (bone scan and CT). Recently, a large study compared ${ }^{68}$ Ga-PSMA PET/CT with CT and bone scan in primary staging of high risk PCa [27]. The study showed that ${ }^{68} \mathrm{Ga}-\mathrm{PSMA}$ PET/CT was superior as compared to conventional imaging, with a higher overall accuracy (92\% vs. 65\%), sensitivity (85\% vs. 38\%) and specificity (98\% vs. 91\%). Furthermore, ${ }^{68} \mathrm{GaPSMA}$ PET/CT also led to more frequent changes in treatment plan. In our study, we investigated if the imaging modality used for primary staging affected the detection rate of ${ }^{68} \mathrm{Ga}$-PSMA PET/CT at biochemical recurrence. Though different detection rates were observed, the median PSA of the patients with ${ }^{68} \mathrm{Ga}$-PSMA PET/CT for primary staging was $0.3 \mathrm{ng} / \mathrm{mL}$ compared with $1 \mathrm{ng} / \mathrm{mL}$ in the group of patients without ${ }^{68} \mathrm{Ga}-\mathrm{PSMA}$ PET/CT for primary staging (staging with other imaging modalities like bone scan and CT). While imaging modality for primary staging at first seemed to influence the detection rate of ${ }^{68}$ Ga-PSMA PET/CT in recurrent disease, the impact disappeared when stratifying for PSA. The non-balanced group sizes could have influenced the findings, but it is important to underline that this observation says nothing about the performance of ${ }^{68} \mathrm{Ga}$-PSMA PET/CT vs. conventional imaging as primary staging modality. Hence, the detection rate seemed to be independent of any primary staging and perioperative characteristics, resulting in no new predictors of the ${ }^{68} \mathrm{Ga}-\mathrm{PSMA}$ PET/CT detection rate being identified.

In agreement with multiple other studies $[9,17,28,29]$, we found that the detection rate was highly associated with increasing pre-PET PSA. In patients with PSA levels below $1 \mathrm{ng} / \mathrm{mL}$, the relapse was only detected in approximately one-third of the patients, whereas the detection rate approached $100 \%$ at PSA levels above $5 \mathrm{ng} / \mathrm{mL}$. As seen in Figure 2, increasing PSA did not only affect the detection rate, but also increased the frequency of dissemination to multiple locations as well. These findings are in agreement with the findings by Fendler et al. [17]. The low detection rate at low PSA levels is probably explained by the lower limit of detection for ${ }^{68} \mathrm{Ga}-\mathrm{PSMA}$ PET/CT, meaning that micro metastatic deposits of PSMA expressing cells remain undetected by ${ }^{68} \mathrm{Ga}-\mathrm{PSMA}$ PET/CT [23]. Patient cases of detected relapse are provided in Figure 3. 


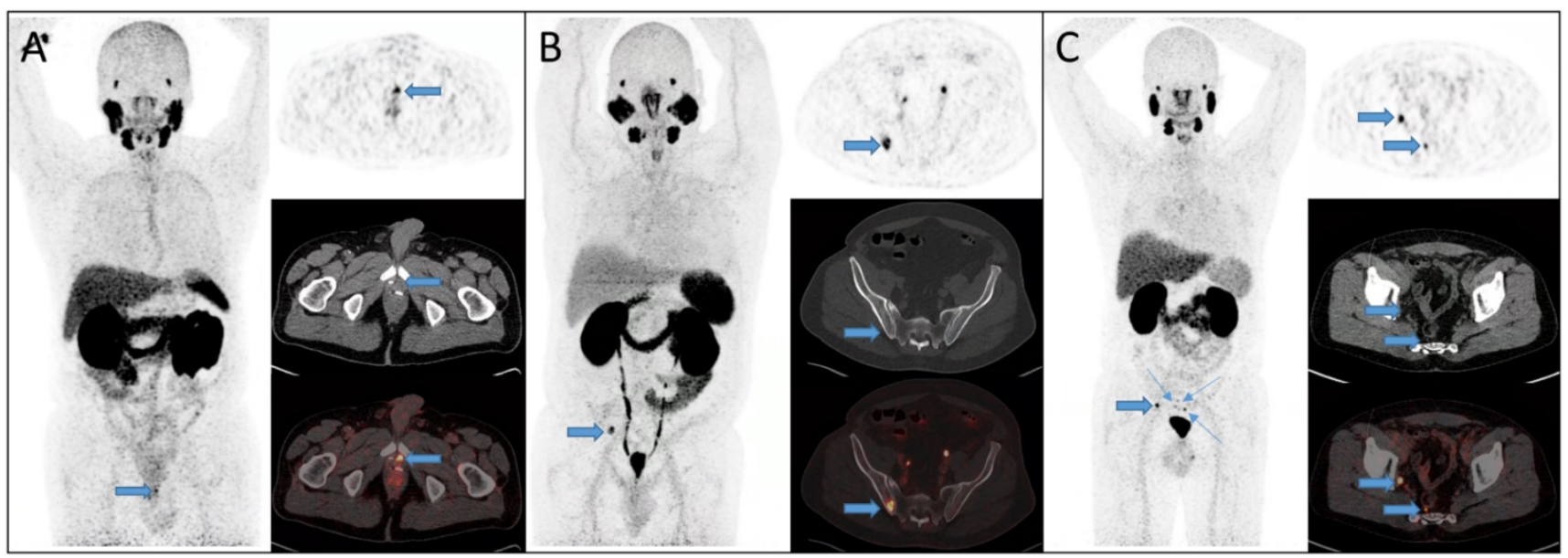

Figure 3. Patients with detected relapse on ${ }^{68}$ Ga-PSMA PET/CT. (A) A 75 year-old man with biochemical recurrence (PSA $=0.5 \mathrm{ng} / \mathrm{mL}$ ) seven years after radical prostatectomy. Local relapse was visualized on ${ }^{68} \mathrm{Ga}$-PSMA PET/CT just anterior to the surgical clips (arrows). Subsequently, radiation therapy was performed on the relapse, and now four years later the patient has unmeasurably low PSA. (B) A 67 year-old male with biochemical recurrence (PSA $=5 \mathrm{ng} / \mathrm{mL}$ ) after salvage radiation therapy. A bone metastasis in the right iliac bone was found (arrows). Subsequently the patient underwent stereotactic radiation therapy of the metastasis with currently two years free of progression. (C) A 59 year-old man with biochemical recurrence (PSA $=1.6 \mathrm{ng} / \mathrm{mL}$ ) three years after radical prostatectomy. ${ }^{68} \mathrm{Ga}-\mathrm{PSMA} \mathrm{PET} / \mathrm{CT}$ showed small pelvic and mesorectal lymph node metastases (arrows).

The detection rates from this study seemed slightly lower than that of other studies. Yet, a large variation between different studies can be observed in the low PSA range [9]. In this study, the equivocal scans $(n=12)$ were pooled with the patients with negative scans, thus perhaps underestimating the detection rate. It is also worth noticing that subdividing the $<0.5 \mathrm{ng} / \mathrm{mL}$ stratum into $0-0.19$ and $0.20-0.49 \mathrm{ng} / \mathrm{mL}$ had an impact on the detection rate (33\% and $45 \%$, respectively) [9], which is why differences in mean PSA in this stratum can affect the detection rate between studies.

The detection rate in this study was $98.8 \%$ in the RT patients and thereby markedly higher than in the RP patients (44.4\%). However, due to differences in the definition of BCR, patients treated with RT by definition have PSA levels larger than that of patients treated with RP (median $4.9 \mathrm{ng} / \mathrm{mL}$ and $0.4 \mathrm{ng} / \mathrm{mL}$, RT and RP, respectively). The difference in the detection rate can for that reason presumably be ascribed to these differences in PSA.

The retrospective nature represents a primary limitation to the current study, as it can imply potential bias. Additionally, the lack of histopathological verification of all PSMA avid lesions on PET provides uncertainty regarding the results of the scans. The relatively small sample size may affect the lack of significance in the association of several of the tested characteristics and the detection rate of ${ }^{68} \mathrm{Ga}$-PSMA PET /CT.

Androgen deprivation therapy (ADT) as a possible predictor of the detection rate has been discussed [28,30]. Preclinical and clinical data indicate that PSMA expression is increased during ADT [31], especially shortly after initiation of ADT, but the clinical impact of ADT on ${ }^{68} \mathrm{GA}-\mathrm{PSMA}$ PET/CT performance requires further study, as mentioned in the guidelines [15]. In our study, information regarding ADT as well as other kinds of treatment between RP or RT and ${ }^{68} \mathrm{Ga}$-PSMA PET/CT for recurrent disease was unavailable in some medical records. Thus, information on ADT was not included in the study, and no subanalysis on ADT was performed. However, we did not expect that the ${ }^{68} \mathrm{Ga}-\mathrm{PSMA} P E T / C T$ scans were performed shortly after the beginning of possible ADT. It is well-known that PSA velocity and PSA doubling time are correlated to ${ }^{68} \mathrm{Ga}$-PSMA PET/CT detection rate at biochemical recurrence, however, information regarding PSA velocity and PSA doubling time has not been taken into account in this study due to unavailability in several patients. 


\section{Conclusions}

Of 189 PCa patients with biochemical recurrence, the relapse was detected on ${ }^{68} \mathrm{Ga}-$ PSMA PET/CT in 103 patients (54.5\%). No new predictors of the ${ }^{68} \mathrm{Ga}-\mathrm{PSMA}$ PET/CT detection rate were identified, as none of the clinical variables acquired during primary staging, pathology reports from prostatectomies, nor imaging modality during primary staging affected the detection rate of ${ }^{68} \mathrm{Ga}$-PSMA PET/CT.

The detection rate of ${ }^{68} \mathrm{Ga}$-PSMA PET/CT was strongly dependent on PSA at recurrence $(p<0.05)$ with increased detection rates, with increasing PSA in agreement with existent literature.

Author Contributions: The study conception and design were mainly contributed by M.T.C., M.R.J. and K.B., but K.D.S., M.B. and S.K. also contributed with critical input to study design. S.K. identified the patients in the database. Statistical analyses were performed by M.T.C. The manuscript was drafted by M.T.C. All authors have read and agreed to the published version of the manuscript.

Funding: This research received no external funding.

Institutional Review Board Statement: This retrospective study was approved by the institutional review board (Central Denmark Region Committees on Health Research Ethics). (Approve code 1-10-72-361-18, 2 June 2019).

Informed Consent Statement: Patient consent was waived due to retrospective study design, and with approval from Ethical Committee/Institutional review board.

Data Availability Statement: Most data supporting the reported results are provided in the paper and further data are stored by the authors.

Conflicts of Interest: The authors declare no conflict of interest.

\section{References}

1. Bray, F.; Ferlay, J.; Soerjomataram, I.; Siegel, R.L.; Torre, L.A.; Jemal, A. Global cancer statistics 2018: GLOBOCAN estimates of incidence and mortality worldwide for 36 cancers in 185 countries. CA Cancer J. Clin. 2018, 68, 394-424. [CrossRef]

2. Cornford, P.; Bellmunt, J.; Bolla, M.; Briers, E.; De Santis, M.; Gross, T.; Henry, A.M.; Joniau, S.; Lam, T.B.; Mason, M.D.; et al. EAU-ESTRO-SIOG Guidelines on Prostate Cancer. Part II: Treatment of Relapsing, Metastatic, and Castration-Resistant Prostate Cancer. Eur. Urol. 2017, 71, 630-642. [CrossRef] [PubMed]

3. Mottet, N.; Cornford, P.; van den Bergh, R.C.N.; Briers, E.; de Santis, M.; Fanti, S.; Gillessen, S.; Grummet, J.; Henry, A.M.; Lam, T.B.; et al. Prostate Cancer. 2020. Available online: https:/ / uroweb.org/guideline/prostate-cancer/\#6_3 (accessed on 16 November 2021).

4. Lütje, S.; Heskamp, S.; Cornelissen, A.S.; Poeppel, T.D.; van den Broek, S.A.; Rosenbaum-Krumme, S.; Bockisch, A.; Gotthardt, M.; Rijpkema, M.; Boerman, O.C. PSMA Ligands for Radionuclide Imaging and Therapy of Prostate Cancer: Clinical Status. Theranostics 2015, 5, 1388-1401. [CrossRef]

5. De Visschere, P.J.L.; Standaert, C.; Fütterer, J.J.; Villeirs, G.M.; Panebianco, V.; Walz, J.; Maurer, T.; Hadaschik, B.A.; Lecouvet, F.E.; Giannarini, G.; et al. A Systematic Review on the Role of Imaging in Early Recurrent Prostate Cancer. Eur. Urol. Oncol. 2019, 2, 47-76. [CrossRef]

6. Zhou, J.; Gou, Z.; Wu, R.; Yuan, Y.; Yu, G.; Zhao, Y. Comparison of PSMA-PET/CT, choline-PET/CT, NaF-PET/CT, MRI, and bone scintigraphy in the diagnosis of bone metastases in patients with prostate cancer: A systematic review and meta-analysis. Skelet. Radiol. 2019, 48, 1915-1924. [CrossRef]

7. Janssen, J.C.; Meißner, S.; Woythal, N.; Prasad, V.; Brenner, W.; Diederichs, G.; Hamm, B.; Makowski, M.R. Comparison of hybrid ${ }^{(68)}$ Ga-PSMA-PET/CT and (99m)Tc-DPD-SPECT/CT for the detection of bone metastases in prostate cancer patients: Additional value of morphologic information from low dose CT. Eur. Radiol. 2018, 28, 610-619. [CrossRef]

8. Schwenck, J.; Rempp, H.; Reischl, G.; Kruck, S.; Stenzl, A.; Nikolaou, K.; Pfannenberg, C.; la Fougere, C. Comparison of ${ }^{(68)}$ Ga-labelled PSMA-11 and (11)C-choline in the detection of prostate cancer metastases by PET/CT. Eur. J. Nucl. Med. Mol. Imaging 2017, 44, 92-101. [CrossRef] [PubMed]

9. Perera, M.; Papa, N.; Roberts, M.; Williams, M.; Udovicich, C.; Vela, I.; Christidis, D.; Bolton, D.; Hofman, M.S.; Lawrentschuk, N.; et al. Gallium-68 Prostate-Specific Membrane Antigen Positron Emission Tomography in Advanced Prostate Cancer-Updated Diagnostic Utility, Sensitivity, Specificity, and Distribution of Prostate-Specific Membrane Antigen-Avid Lesions: A Systematic Review and Meta-Analysis. Eur. Urol. 2020, 77, 403-417. [PubMed]

10. Beresford, M.J.; Gillatt, D.; Benson, R.J.; Ajithkumar, T. A systematic review of the role of imaging before salvage radiotherapy for post-prostatectomy biochemical recurrence. Clin. Oncol. (R. Coll. Radiol.) 2010, 22, 46-55. [CrossRef] [PubMed] 
11. Han, S.; Woo, S.; Kim, Y.J.; Suh, C.H. Impact of ${ }^{(68)}$ Ga-PSMA PET on the Management of Patients with Prostate Cancer: A Systematic Review and Meta-Analysis. Eur. Urol. 2018, 74, 179-190. [CrossRef]

12. Harris, P.A.; Taylor, R.; Minor, B.L.; Elliott, V.; Fernandez, M.; O’Neal, L.; McLeod, L.; Delacqua, G.; Delacqua, F.; Kirby, J.; et al. The REDCap consortium: Building an international community of software platform partners. J. Biomed. Inform. 2019, 95, 103208. [CrossRef]

13. Harris, P.A.; Taylor, R.; Thielke, R.; Payne, J.; Gonzalez, N.; Conde, J.G. Research electronic data capture (REDCap)—A metadatadriven methodology and workflow process for providing translational research informatics support. J. Biomed. Inform. 2009, 42, 377-381. [CrossRef] [PubMed]

14. Epstein, J.I.; Egevad, L.; Amin, M.B.; Delahunt, B.; Srigley, J.R.; Humphrey, P.A. The 2014 International Society of Urological Pathology (ISUP) Consensus Conference on Gleason Grading of Prostatic Carcinoma: Definition of Grading Patterns and Proposal for a New Grading System. Am. J. Surg. Pathol. 2016, 40, 244-252. [CrossRef] [PubMed]

15. Fendler, W.P.; Eiber, M.; Beheshti, M.; Bomanji, J.; Ceci, F.; Cho, S.; Giesel, F.; Haberkorn, U.; Hope, T.A.; Kopka, K.; et al. ${ }^{(68)}$ Ga-PSMA PET/CT: Joint EANM and SNMMI procedure guideline for prostate cancer imaging: Version 1.0. Eur. J. Nucl. Med. Mol. Imaging 2017, 44, 1014-1024. [CrossRef] [PubMed]

16. Eiber, M.; Herrmann, K.; Calais, J.; Hadaschik, B.; Giesel, F.L.; Hartenbach, M.; Hope, T.A.; Reiter, R.; Maurer, T.; Weber, W.A.; et al. Prostate Cancer Molecular Imaging Standardized Evaluation (PROMISE): Proposed miTNM Classification for the Interpretation of PSMA-Ligand PET/CT. J. Nucl. Med. 2018, 59, 469-478. [CrossRef] [PubMed]

17. Fendler, W.P.; Calais, J.; Eiber, M.; Flavell, R.R.; Mishoe, A.; Feng, F.Y.; Nguyen, H.G.; Reiter, R.E.; Rettig, M.B.; Okamoto, S.; et al. Assessment of 68Ga-PSMA-11 PET Accuracy in Localizing Recurrent Prostate Cancer: A Prospective Single-Arm Clinical Trial. JAMA Oncol. 2019, 5, 856-863. [CrossRef]

18. Hope, T.A.; Eiber, M.; Armstrong, W.R.; Juarez, R.; Murthy, V.; Lawhn-Heath, C.; Behr, S.C.; Zhang, L.; Barbato, F.; Ceci, F.; et al. Diagnostic Accuracy of 68Ga-PSMA-11 PET for Pelvic Nodal Metastasis Detection Prior to Radical Prostatectomy and Pelvic Lymph Node Dissection: A Multicenter Prospective Phase 3 Imaging Trial. JAMA Oncol. 2021, 7, 1635-1642. [CrossRef]

19. Mei, R.; Farolfi, A.; Castellucci, P.; Nanni, C.; Zanoni, L.; Fanti, S. PET/CT Variants and Pitfalls in Prostate Cancer: What You Might See on PET and Should Never Forget. Semin. Nucl. Med. 2021, 51, 621-632. [CrossRef]

20. D'Amico, A.V.; Whittington, R.; Malkowicz, S.B.; Schultz, D.; Blank, K.; Broderick, G.A.; Tomaszewski, J.E.; Renshaw, A.A.; Kaplan, I.; Beard, C.J.; et al. Biochemical outcome after radical prostatectomy, external beam radiation therapy, or interstitial radiation therapy for clinically localized prostate cancer. JAMA 1998, 280, 969-974. [CrossRef]

21. Franzese, E.; Falco, S.; Laterza, M.M.; Montella, L.; Facchini, S.; Liguori, C.; Coppola, P.; Diessa, Y.; Berretta, M.; Pisconti, S.; et al. The use of ${ }^{(68)}$ Ga prostate-specific membrane antigen PET-CT in prostate cancer: Diagnostic challenges and therapeutic opportunities. Future Sci. OA 2021, 7, Fso705. [CrossRef]

22. Ross, J.S.; Sheehan, C.E.; Fisher, H.A.; Kaufman, R.P.; Kaur, P., Jr.; Gray, K.; Webb, I.; Gray, G.S.; Mosher, R.; Kallakury, B.V.S. Correlation of primary tumor prostate-specific membrane antigen expression with disease recurrence in prostate cancer. Clin. Cancer Res. 2003, 9, 6357-6362.

23. Klingenberg, S.; Jochumsen, M.R.; Ulhøi, B.P.; Fredsøe, J.; Sørensen, K.D.; Borre, M.; Bouchelouche, K. ${ }^{(68)}$ Ga-PSMA PET/CT for primary NM staging of high-risk prostate cancer. J. Nucl. Med. 2020, 62, 214-220. [CrossRef] [PubMed]

24. Uprimny, C.; Kroiss, A.S.; Decristoforo, C.; Fritz, J.; von Guggenberg, E.; Kendler, D.; Scarpa, L.; di Santo, G.; Roig, L.G.; Maffey-Steffan, J.; et al. ${ }^{(68)}$ Ga-PSMA-11 PET/CT in primary staging of prostate cancer: PSA and Gleason score predict the intensity of tracer accumulation in the primary tumour. Eur. J. Nucl. Med. Mol. Imaging 2017, 44, 941-949. [CrossRef]

25. Jochumsen, M.R.; Sörensen, J.; Tolbod, L.P.; Pedersen, B.G.; Frøkiær, J.; Borre, M.; Bouchelouche, K. Potential synergy between PSMA uptake and tumour blood flow for prediction of human prostate cancer aggressiveness. EJNMMI Res. 2021, 11, 12. [CrossRef]

26. Vinsensia, M.; Chyoke, P.L.; Hadaschik, B.; Holland-Letz, T.; Moltz, J.; Kopka, K.; Rauscher, I.; Mier, W.; Schwaiger, M.; Haberkorn, U.; et al. ${ }^{(68)}$ Ga-PSMA PET/CT and Volumetric Morphology of PET-Positive Lymph Nodes Stratified by Tumor Differentiation of Prostate Cancer. J. Nucl. Med. 2017, 58, 1949-1955. [CrossRef]

27. Hofman, M.S.; Lawrentschuk, N.; Francis, R.J.; Tang, C.; Vela, I.; Thomas, P.; Rutherford, N.; Martin, J.M.; Frydenberg, M.; Shakher, R.; et al. Prostate-specific membrane antigen PET-CT in patients with high-risk prostate cancer before curative-intent surgery or radiotherapy (proPSMA): A prospective, randomised, multicentre study. Lancet 2020, 395, 1208-1216. [CrossRef]

28. Afshar-Oromieh, A.; Holland-Letz, T.; Giesel, F.L.; Kratochwil, C.; Mier, W.; Haufe, S.; Debus, N.; Eder, M.; Eisenhut, M.; Schäfer, M.; et al. Diagnostic performance of ${ }^{(68)}$ Ga-PSMA-11 (HBED-CC) PET/CT in patients with recurrent prostate cancer: Evaluation in 1007 patients. Eur. J. Nucl. Med. Mol. Imaging 2017, 44, 1258-1268. [CrossRef] [PubMed]

29. Rauscher, I.; Düwel, C.; Haller, B.; Rischpler, C.; Heck, M.M.; Gschwend, J.E.; Schwaiger, M.; Maurer, T.; Eiber, M. Efficacy, Predictive Factors, and Prediction Nomograms for ${ }^{(68)}$ Ga-labeled Prostate-Specific Membrane Antigen-Ligand Positron-Emission Tomography/Computed Tomography in Early Biochemical Recurrent Prostate Cancer After Radical Prostatectomy. Eur. Urol. 2018, 73, 656-661. [CrossRef] 
30. Eiber, M.; Maurer, T.; Souvatzoglou, M.; Beer, A.J.; Ruffani, A.; Haller, B.; Graner, F.-P.; Kübler, H.; Haberhorn, U.; Eisenhut, M.; et al. Evaluation of Hybrid ${ }^{68}$ Ga-PSMA Ligand PET/CT in 248 Patients with Biochemical Recurrence After Radical Prostatectomy. J. Nucl. Med. 2015, 56, 668-674. [CrossRef] [PubMed]

31. Einspieler, I.; Rauscher, I.; Düwel, C.; Krönke, M.; Rischpler, C.; Habl, G.; Dewes, S.; Ott, A.; Wester, H.-J.; Schwaiger, M.; et al. Detection Efficacy of Hybrid ${ }^{(68)}$ Ga-PSMA Ligand PET/CT in Prostate Cancer Patients with Biochemical Recurrence after Primary Radiation Therapy Defined by Phoenix Criteria. J. Nucl. Med. 2017, 58, 1081-1087. [CrossRef] [PubMed] 